\title{
Werkvertragsunternehmen: Outsourcing auf dem Betriebsgelände
}

HARTMUT KLEIN-SCHNEIDER, KAI BEUTLER

\section{Werkverträge - „alter Hut“ oder neue Strategie?}

Wir beobachten aktuell einen Strategiewechsel in Industrieund Dienstleistungsunternehmen: Tätigkeiten und Produktionsschritte, die bislang durch die Stammbeschäftigten des Unternehmens ausgeführt worden sind, werden an ein anderes, zweites Unternehmen vergeben. Dieses zweite Unternehmen wird mit einem Werkvertrag beauftragt und führt die vereinbarten Tätigkeiten mit eigenem Personal, aber an den Maschinen und auf dem Betriebsgelände des ersten, beauftragenden Unternehmens aus. Zielsetzung dieser Konstruktion scheint es zu sein, tarifvertragliche, arbeitsund mitbestimmungsrechtliche Ansprüche der (Stamm-) Beschäftigten des Industrieunternehmens zu umgehen. Solche Werkvertragsunternehmen haben sich nicht nur im Bereich industrieller Produktion etabliert, sondern auch bei privaten und öffentlichen Dienstleistungen, u.a. im Handel und in Krankenhäusern.

Was ist das Neue daran? Worin unterscheiden sich diese Werkverträge von Leiharbeit, von „Outsourcing“ und von den Werkverträgen, wie sie schon seit Jahren in der Wirtschaft vereinbart werden?

Werkverträge wurden bisher vor allem dann eingesetzt, wenn ein Unternehmen für ein bestimmtes Produkt, ein Gewerk oder eine Leistung eigene Kapazitäten und fachkompetentes Personal nicht vorhalten will. D. h. die Unternehmen kaufen Produkte und Leistungen ein, die sie nur gelegentlich benötigen und deren Erstellung sie nicht zum Kernkompetenzbereich ihres Unternehmens zählen. Beispiele hierfür sind die vielen verschiedenen Gewerke beim Bau und Ausbau von Gebäuden oder Industrieanlagen, Kantinen, IT-Produkte und IT-Service. Dieser Typus der Werkvertragsvergabe wird auch weiterhin praktiziert. Die im Mittelpunkt der folgenden Betrachtung stehenden „neuen“ Werkverträge unterscheiden sich hiervon in dreierlei Hinsicht:

(1) Heute werden zunehmend Werkverträge aufDauer und nicht nur gelegentlich abgeschlossen.

(2) Es werden Werke, Produkte oder Leistungen mit Werkvertrag zugekauft, die bisher zum Kernbereich der eigenen Produktion gehörten.
(3) Die Produkte oder Leistungen werden von dem beauftragten Fremdunternehmen auf dem Betriebsgelände und an Arbeitsplätzen und Maschinen des beauftragenden Unternehmens erstellt.

Ebenso wie Leiharbeiterinnen und Leiharbeiter werden die Arbeitskräfte des Werkvertragsunternehmens auf dem Betriebsgelände und an den Anlagen des beauftragenden Unternehmens tätig. Jedoch: Während Leiharbeit nach intensiven Bemühungen der Gewerkschaften ansatzweise wieder in rechtlich regulierten Formen eingesetzt wird, also „rereguliert“ worden ist, gilt Gleiches für die Beschäftigung in Werkvertragsunternehmen nicht. Es greift weder der Grundsatz des „Equal Pay“, also gleicher Lohn für gleiche Arbeit, noch haben die Beschäftigten des Werkvertragsunternehmens Zugang zum Betriebsrat des beauftragenden Unternehmens; und sie haben auch sonst keinerlei Ansprüche gegenüber dem beauftragenden Unternehmen.

Ähnlichkeiten gibt es zwischen der Beauftragung von Werkvertragsunternehmen und dem „Outsourcing“, dass wir seit ca. zwei Jahrzehnten kennen. Mit „Outsourcing“ ist die Vergabe bestimmter Produkte oder Gewerke von Industrie- und Dienstleistungsunternehmen an ein „Fremdunternehmen“ gemeint, das diese Produkte oder Gewerke besser oder effizienter ausführen kann. ${ }^{\bullet}$ Die hier zur Diskussion stehenden Werkverträge unterscheiden sich vom „Outsourcing“ jedoch darin, dass Gewerke oder Tätigkeiten nicht aus dem Betriebsgelände hinaus verlagert werden. Deshalb auch die Bezeichnung als „Onsite-Werkvertragsunternehmen“. Eine Verbesserung der Effizienz und Qualität der Erstellung der Gewerke durch die Beauftragung eines Werkvertragsunternehmens wird nicht allein (ggf. auch gar nicht) angestrebt; treibendes Motiv scheint vielmehr zu sein, dass die Lohnkosten stark abgesenkt werden können. Das Werkvertragsunternehmen bietet die geforderte Leistung zu niedrigeren Kosten an - der beim Einsatz von Stammbeschäftigten nicht zu erzielen wäre.

Festzustellen ist also eine neue Entwicklung: Werkverträge werden abgeschlossen, um Tätigkeiten aus dem Kern-

\footnotetext{
Vgl. Müller, H. E./Prangenberg, A. (1997): Outsourcing Management, Frankfurt a. M.
} 
bereich eines Unternehmens von einem zweiten, einem Fremdunternehmen ausführen zu lassen. Dabei werden die Tätigkeiten an den gleichen Arbeitsplätzen und auf dem gleichen Betriebsgelände wie vorher ausgeübt. Das Besondere ist die Kombination von „Outsourcing“ auf dem Werksgelände mit der Vergabe in Form eines Werkvertrages.

\section{Worin liegt das Problem?}

Die Bundesregierung sieht auf Anfrage der Bundestagsfraktion „Die Linke“ keine Hinweise auf eine „weit verbreitete, systematisierte missbräuchliche Nutzung von Werkverträgen zur Umgehung von tariflichen oder arbeitsrechtlichen Standards. “? Eine gänzlich andere Sichtweise zeigt sich allerdings in Erfahrungsberichten von Beschäftigten solcher Werkvertragsunternehmen, in Diskussionen auf gewerkschaftlichen Branchentagungen und in ersten systematischen Untersuchungen. ${ }^{3}$ Demzufolge werden im Zuge der Beauftragung von Werkvertragsunternehmen tarifvertraglich vereinbarte Entgelt- und Arbeitsbedingungen der Stammbeschäftigten tatsächlich unterlaufen und damit letztlich auch infrage gestellt. Die Beschäftigten des Werkvertragsunternehmens wiederum haben, selbst wenn sie die gleiche Arbeit leisten wie Stammbeschäftigte des Unternehmens, in dem sie eingesetzt sind, keinen Anspruch auf gleiche Bezahlung. Ihr Vertragspartner ist einzig das Werkvertragsunternehmen. Bei einer möglichen Kündigung des Werkvertrags greift für die Beschäftigten des Werkvertragsunternehmens kein Kündigungsschutz. Sie haben in solch einem Fall kaum noch rechtliche Handlungsmöglichkeiten, da das Werkvertragsunternehmen nach Kündigung des Werkvertrages selbst nicht mehr über Beschäftigungsmöglichkeiten verfügt. Außerdem fehlt es in Werkvertragsunternehmen häufig an einer gewählten Interessenvertretung. Und selbst, wenn die Beschäftigten eines Werkvertragsunternehmens einen eigenen Betriebsrat wählen, hat der nur geringe Möglichkeiten, mit den Verantwortlichen des beauftragenden Unternehmens über die Arbeitsbedingungen zu verhandeln. Der Betriebsrat des beauftragenden Unternehmens hat kein Mandat zur Interessenvertretung der Beschäftigten des Werkvertragsunternehmens. In vielen Fällen werden Arbeitsschutzvorschriften von Werkvertragsunternehmen nicht eingehalten, die Beschäftigten werden nicht ausreichend unterwiesen, sie bekommen nicht die gleiche persönliche Arbeitsschutzausrüstung wie die Stammbeschäftigten, die Tätigkeiten von Stammbeschäftigten und Werkvertragsbeschäftigten werden unter dem Aspekt des Arbeitsschutzes unzureichend koordiniert. Die Schlechterstellungen hinsichtlich Arbeitsentgelt, Arbeitsbedingungen, Arbeitsschutz und Beschäftigungssicherheit sind evident.

Nicht wenige dieser Werkverträge erweisen sich bei genauer Betrachtung als versteckte Leiharbeit, weil das beauftragte Unternehmen über keinen unternehmerischen Handlungsspielraum verfügt, keine Ergebnisverantwortung übernimmt und für Fehlleistungen nicht haftet. ${ }^{\oplus}$
Negative Auswirkungen im Zuge der Beauftragung von Werkvertragsunternehmen sind jedoch nicht nur für die Beschäftigten zu befürchten, sondern auch für das beauftragende Unternehmen. Bei jeder Auftragsvergabe bleibt eine Unsicherheit, ob Qualitätsanforderungen des beauftragenden Unternehmens erfüllt und Liefertermine eingehalten werden können. Gegebenenfalls verliert das beauftragende Unternehmen Qualifikationen und damit spezifische Wettbewerbsvorteile.

Über die Entgelt- und Arbeitsbedingungen der Beschäftigten der Werkvertragsunternehmen liegen bislang nur wenige belastbare empirische Daten vor. Verallgemeinerungen verbieten sich auf dem aktuellen Wissensstand. Vermutlich sind die Arbeits- und Entgeltbedingungen sehr unterschiedlich, je nach Qualifikation und Spezialisierung der benötigten Arbeitskräfte und je nach Zielsetzung der Beauftragung mit Werkvertrag. Die vorliegenden Forschungsbefunde geben Anlass, auf betrieblicher, tariflicher und gesetzgeberischer Ebene Verbesserungen der Arbeitsbedingungen der in den Werkvertragsunternehmen Tätigen einzufordern. Das noch neue Phänomen der Werkvertragsunternehmen muss weiter beobachtet werden. Erste qualitative Befunde bedürfen der Absicherung durch respräsentative Untersuchungen.

\section{Ausmaß und Entwicklung des Einsatzes von Beschäftigten über Werkverträge}

Auch Ausmaß und Entwicklung des Einsatzes von Werkvertragsunternehmen für die Kernaufgaben und -produktionsbereiche von Industrie- oder Dienstleistungsunternehmen sind bisher nur lückenhaft erforscht. D. h. es gibt auch hier nur wenige repräsentative Daten; es gibt jedoch erste empirische Untersuchungen ${ }^{\ominus}$ und gutachterliche

(2) Bundestagsdrucksache BT 17/6714 vom 01.08.2011.

(3) Vgl. hierzu das Projekt „Handlungskompetenz von Betriebsräten beim Auslagern von betrieblichen Aufgaben an Werkvertragsunternehmen auf dem Betriebsgelände und Einsatz von Onsite-Werkvertragsarbeitnehmer/innen (oWAN-BR)", gefördert von der Hans-Böckler-Stiftung, durchgeführt von bsb $\mathrm{GmbH}$, verantwortlich: Kai Beutler.

(4) Vgl. Schüren, P. (2012): Zur rechtlichen Situation und zur Abgrenzung des Einsatzes von Werkvertragsunternehmen, Vortrag gehalten auf der Fachtagung „Werkvertragsunternehmen" am 26./27.11. in Frankfurt a. M., http://www. boeckler.de/pdf/v_2012_11_27_schueren.pdf.

(5) Vgl. IG Metall Vorstand Zweigbüro (2011): Beschäftigung am Rande. Auswertung der Umfrage „Bestandsaufnahme zum Umfang von Werk- und Dienstleistungsverträgen in der Stahlbranche"; Beutler, K. (2012): Was wissen wir über Entwicklung, Ausmaß und Auswirkungen derTätigkeit von Werkvertragsunternehmen? Vortrag auf der Fachtagung „Werkvertragsunternehmen“ am 26./27.11. in Frankfurt a. M.; http://www.boeckler.de/pdf/v_2012_11_26_beutler.pdf. 
Einschätzungen und Bewertungen, ${ }^{\odot}$ die auf die Tiefe des Problems verweisen.

Die Medien haben bereits eine Vielzahl von Fallbeispielen veröffentlicht. ${ }^{0}$ Die IG Metall und Gewerkschaft Nahrung, Genuss, Gaststätten (NGG) haben in einigen Branchen Betriebsräte befragt. Werkverträge werden auf vielen gewerkschaftlichen (Branchen-)Tagungen diskutiert und sind Gegenstand von Aktionen und Beschlüssen.

Befragungen von Betriebsratsmitgliedern auf gewerkschaftlichen Branchenkonferenzen zeigen eine große Spannweite: Demnach sind in der Schmiedeindustrie Fremdunternehmen mit Werkverträgen nur eine Randerscheinung. Dagegen gibt es in den Schlachthöfen nur noch gut $20 \%$ fest angestellte Stammbeschäftigte. Hinzu kommen 5 \% Leiharbeitnehmer und 75 \% Beschäftigte von Werkvertragsunternehmen. In der Getränkeindustrie werden ca. $10 \%$ der Arbeitskräfte über Werkvertragsunternehmen beschäftigt, in der Zuckerindustrie sind es fast $20 \%$, in den Werften ebenfalls knapp $20 \%$ und in der Fleischindustrie sogar ca. 35 \% der Arbeitnehmerinnen und Arbeitnehmer, die über Werkvertragsunternehmen beschäftigt werden. ${ }^{\circledR}$ Diese Werte sind in einzelnen Befragungen gewonnen worden, sie sind damit nicht repräsentativ, zeigen aber eine Entwicklungsrichtung an.

Gleiches gilt mit Blick auf die Stahlindustrie, wo durchschnittlich $8 \%$ der Arbeitskräfte bei einem Werkvertragsunternehmen beschäftigt sind (5.172 von 62.000 Beschäftigten). ${ }^{\ominus}$ In acht der befragten Betriebe finden wir einen Anteil der Werkvertragsarbeitnehmer von $15 \%$ bis maximal $34 \%$ an den Beschäftigten insgesamt. Die Entwicklung der Beschäftigung über Werkvertragsunternehmen können wir beispielhaft in einer Fallstudie von Beutler ${ }^{(1)}$ erkennen: In diesem Betrieb ist der Anteil der Stammbeschäftigten seit 2006 um ca. 20 Prozentpunkte gesunken. Dabei ist die Leiharbeit zunächst von knapp $5 \%$ auf $14 \%$ gestiegen, inzwischen aber auf weniger als $2 \%$ gefallen. Seit 2008, parallel zum Absinken des Anteils der Leiharbeit, ist der Anteil der Werkvertragsarbeit von null auf 24,8\% der Beschäftigten angestiegen.

Die Komplexität der Beschäftigungsverhältnisse lässt sich exemplarisch am Einsatz von Leiharbeit und Werkvertragsarbeit bei Porsche und bei BMW in Leipzig zeigen: Nur noch ein Bruchteil der Arbeitskräfte dieser beiden Automobilbetriebe verfügt über einen unbefristeten Arbeitsvertrag mit der Porsche AG oder der BMW AG. Der Industriedienstleister ThyssenKrupp Automotive ist per Werkvertrag beauftragt, eine Reihe von Produktionsschritten mit eigenem Personal zu leisten. Die ThyssenKrupp Automotive beschäftigt selbst nur 34 Mitarbeiter und Mitarbeiterinnen vor Ort, davon zehn Leiharbeitsbeschäftigte. Darüber hinaus setzt sie über 350 Beschäftigte der WISAG über einen Arbeitnehmerüberlassungsvertrag ein und leiht weitere 120 Arbeitskräfte von sechs Verleihfirmen aus. ${ }^{\text {(1) }}$

Diese Daten können, wie bereits erwähnt, auf die Industrie und auf die Wirtschaft insgesamt nicht hochgerechnet werden. Sie verweisen dennoch auf ein wachsendes Problem: Normalarbeitsverhältnisse, die sozialen und rechtlichen Standards entsprechen, werden durch neue Beschäftigungsformen verdrängt, auf die Interessenvertretungen keinen oder kaum Einfluss haben: weder die Betriebsräte der beauftragenden Industrie- und Dienstleistungsunternehmen noch die Betriebsräte der Werkvertragsunternehmen - falls hier überhaupt eine betriebliche Interessenvertretung existiert.

\section{Handlungsnotwendigkeiten und Handlungs- möglichkeiten}

(1) Auf betrieblicher Ebene bedarf es der Unterstützung der Betriebsräte und der Gewerkschaften in der Auseinandersetzung um die Einführung dieser neuen „Personaleinsatzstrategie“. Das Betriebsverfassungsgesetz sieht kein hartes Mitbestimmungsrecht für Fragen der Beauftragung eines Fremdunternehmens vor. Andererseits sind Auswirkungen eines solchen „Einsatzes eines Fremdunternehmens per Werkvertrag“ auf die Beschäftigung im Industrieunternehmen beabsichtigt und deshalb zu erwarten. Ein Betriebsrat tut also gut daran, frühzeitig seine Informationsrechte zu nutzen, um eine Strategie des Unternehmens zu erkennen und diese offensiv zur Diskussion zu stellen. Fragen der Abgrenzung „echter“ Werkverträge von Scheinwerkverträgen und verkappter Leiharbeit, der Personalplanung, der Auswirkungen der Maßnahmen auf die Beschäftigten und der mittel- und langfristigen Entwicklung bieten Ansatzpunkte, um die Beauftragung von Fremdunternehmen über Werkverträge in Gesprächen zwischen Betriebsrat und Unternehmensleitung und im Wirtschaftsausschuss zu thematisie-

(6 Vgl. Waas, B. (2012): Werkvertrag, freier Dienstvertrag und Arbeitsvertrag. Gutachten im Auftrag der Hans-BöcklerStiftung; Däubler, W. (2011): Regulierungsmöglichkeiten im Zusammenhang von Werkverträgen. Expertise erstellt im Auftrag der Bundestagsfraktion Die Linke, Berlin; Koch, A. (2012): Werkverträge in der Arbeitswelt: Otto-BrennerStiftung (Hrsg.), OBS-Arbeitspapier (2).

(7) Vgl. bspw. die tageszeitung vom 20./21.08.2011; Die Zeit online vom 29.08.2011; metallzeitung 1/2012; Gute Arbeit 6/2012; telepolis vom 18.12.2012; Sendung Report aus Mainz am 15.01.2013 im ARD-Fernsehprogramm.

8 Vgl. Beutler, K. (2012), a.a.O. (Fußnote 5).

(9 IG Metall Vorstand Zweigbüro (2011), a.a.O. (Fußnote 5).

(10) Vgl. Beutler, K. (2012), a.a.O.

(11) Graupner, Ch. (2012): Vortrag: „Was wissen wir über Entwicklung, Ausmaß und Auswirkungen derTätigkeit von Werkvertragsunternehmen?", gehalten am 26./27.11. in Frankfurt a. M., http://www.boeckler.de/pdf/v_2012_11_26_ graupner.pdf. 
ren. Erste Handlungshilfen für die betriebliche Ebene liegen vor. ${ }^{(1)}$

(2) Herauszuarbeiten sind die Auswirkungen auf die Beschäftigten und auf das Unternehmen und seine Wettbewerbsfähigkeit. Eine erste Aufgabe besteht darin, zu prüfen, ob alle möglichen Wirkungen auf Kosten, auf Termintreue, auf Produktqualität und auf einen möglichen Qualifikationsverlust bei einer Entscheidung über „Make or Buy“ hinreichend berücksichtigt worden sind. Sind Beschäftigte und Betriebsrat aufgrund der Konjunktur oder des Fachkräftebedarfs in einer starken Position gegenüber der Unternehmensleitung, kann der Betriebsrat sogar versuchen, eine Betriebsvereinbarung über den Ausschluss oder die Begrenzung von Leiharbeit und/oder Werkvertragsarbeit zu verhandeln.

(3) Ist die Entscheidung getroffen, Fremdfirmen per Werkvertrag auf das Unternehmensgelände zu holen, geht es um die Auswahl des zu beauftragenden Unternehmens und um die Organisation der Zusammenarbeit. Das Arbeitssicherheitsgesetz schreibt vor, dass Arbeiten von Fremdfirmen auf dem Betriebsgelände zur Vermeidung von gegenseitigen Gefährdungen der Beschäftigten des beauftragenden und des beauftragten Unternehmens zu koordinieren sind. Im Arbeitsschutz hat der Betriebsrat verhältnismäßig starke Rechte.

(4) Handlungsmöglichkeiten ergeben sich auf der betrieblichen Ebene weiterhin in Bezug auf die Prüfung, ob es sich hier um „Scheinwerkverträge“ handelt, die tatsächlich bestehende Leiharbeit nur verstecken sollen.

(5) Schließlich kann sich der Betriebsrat mit den Arbeitsbedingungen der Beschäftigten des Fremdunternehmens auseinandersetzen, auch wenn das Betriebsverfassungsgesetz ihm dafür zunächst kein Mandat erteilt.

(6) Bei all diesen Aufgaben und Ansätzen bedürfen die Betriebsräte der Unterstützung der Gewerkschaften.

(7) Zunächst sind die tarifpolitischen Instrumente daraufhin auszurichten, einer ausufernden Fremdvergabe mithilfe von Werkverträgen entgegenzusteuern. Gleichzeitig müssen sich die Gewerkschaften um die Organisation der Beschäftigten dieser neuen Industriedienstleister oder Werkvertragsunternehmen bemühen.

(8) Tarifvertragliche Gestaltungsansätze greifen umso effektiver, je mehr man weiß, welche Strategien Unternehmen mit der Beauftragung von Werkvertragsunternehmen verfolgen. Zielen sie ausschließlich darauf, (Flächen-)Tarifverträge zu unterlaufen und kurzfristige Kostenvorteile (Absenkung der Lohnkosten) zu erzielen? Oder geht es darum, Beschäftigungsverhältnisse zu flexibilisieren und sich von arbeits- und mitbestimmungsrechtlichen Verpflichtungen wie dem Kündigungsschutzgesetz zu „befreien“? Wenn die Unternehmen die Strategie der Fremdvergabe auf ihrem Unternehmensgelände auch im Hinblick auf die Produktqualität, die Verfügbarkeit qualifizierter Arbeitskräfte, die Termineinhaltung usw. erfolgreich gestalten können, stellen sich völlig neue Fragen für die Gewerkschaften. Das Industrieverbandssystem, Branchen- und Flächentarifverträge wären dann infrage gestellt. Langfristig droht möglicherweise eine Trennung von Produktion und Beschäftigung mit noch nicht absehbaren Folgen.

(9) Schließlich müssen die Politik, der Gesetzgeber und die Öffentlichkeit für diese Entwicklung sensibilisiert werden, es muss auf den Missbrauch von Werkverträgen zur Umgehung tariflicher und arbeitsrechtlicher Verpflichtungen aufmerksam gemacht werden. Dafür dienen nicht zuletzt auch Untersuchungen und Tagungen der Hans-BöcklerStiftung. Erste Überlegungen zu erforderlichen gesetzgeberischen Maßnahmen liegen bereits vor, ${ }^{\circledR}$ der Deutsche Gewerkschaftsbund hat seine Position veröffentlicht. ${ }^{(4)} \mathrm{Er}$ fordert darin u. a. die Ordnung am Arbeitsmarkt (wieder) herzustellen, Lohndumping durch Werkverträge zu unterbinden und Interessenvertretungen und Mitbestimmungsrechte zu stärken.

\section{Ausblick: Die weitere Entwicklung und Forschungsbedarf}

Der Entwicklungstrend ist offensichtlich: Industrie- und Dienstleistungsunternehmen gehen zunehmend dazu über, Fremdunternehmen per Werkvertrag zu beauftragen, Tätigkeiten und Produktionsschritte zu übernehmen, die bislang vom Stammpersonal ausgeführt worden sind. Die Besonderheit dieser Werkvertragsunternehmen ist, dass die Tätigkeiten nicht etwa ausgelagert werden, sondern im Gegenteil mit deren eigenem Personal an den Anlagen und Arbeitsplätzen des beauftragenden Industrie- oder Dienstleistungsunternehmens ausgeführt werden. Diese Entwicklung kann in einer Linie mit der Zunahme der Leiharbeit in den vergangenen Jahren gesehen werden. Sie kann aber darüber hinaus auch als Reaktion darauf gewertet werden, dass es den Gewerkschaften in jüngster Zeit gelungen

(12) Vgl. IG Metall Vorstand (2011) (Hrsg.): Viel Arbeit am Rand. Werkverträge und Outsourcing: Arbeitshilfe für Betriebsräte; Scheriau, K. M. (2012): Beteiligungsrechte des Betriebsrats bei Personal- und Unternehmensplanung, in: Arbeitsrecht im Betrieb (AiB) 33 (3), S. 154-159.

(13) Vgl. Däubler, W. (2011): Regulierungsmöglichkeiten im Zusammenhang von Werkverträgen. Expertise erstellt im Auftrag der Bundestagsfraktion Die Linke, Berlin.

(44) DGB Bundesvorstand (2012): Positionspapier des DGB Bundesvorstandes gegen die missbräuchliche Nutzung von Werkverträgen, Berlin. 
ist, die Leiharbeit zu „reregulieren“. Werkvertragsunternehmen sind dann - aus Unternehmenssicht - eine alternative Ausweichstrategie. Der Druck des globalisierten Wettbewerbs führt offenbar zu einer immer wieder neuen und verstärkten Suche der Unternehmen nach Wettbewerbsvorteilen auch in Gestaltungsfeldern, die dem Wettbewerb zumindest eine Zeit lang entzogen waren: tarifvertraglich gestaltete Arbeits- und Entgeltbedingungen, Arbeitsrecht, Arbeitsschutzrecht, Beschäftigungsverhältnisse und Mitbestimmung. Die damit erzielten Wettbewerbsvorteile sind teilweise nur von sehr kurzer Dauer. Dennoch wird diese „Strategie“ weiter verfolgt und führt zu Dumping-Löhnen und Dumping-Arbeitsbedingungen in den Industriewie in Schwellen- und Entwicklungsländern. Die bisherige Gegenwehr der Gewerkschaften läuft dieser dynamischen Entwicklung zwangsweise hinterher: Kaum ist ein Problem wie die Regulierung der Leiharbeit scheinbar gelöst, werden neue Problemkonstellationen wie die Etablierung von Werkvertragsunternehmen sichtbar. Die Mitbestimmungs- und Forschungsförderung in der Hans-Böckler-Stiftung versucht, solche Entwicklungen auf allen Ebenen, auf der betrieblichen, der tariflichen und der gesetzgeberischen, rechtzeitig zu erfassen und zu analysieren: vor allem, um gemeinsam mit den betroffenen Akteurinnen und Akteuren der Mitbestimmung Handlungsstrategien entwickeln und umzusetzen zu können. Parallel dazu gilt es, die mittel- und langfristige Entwicklung, hier insbesondere die Perspektiven für die Beschäftigungsverhältnisse insgesamt, zu beobachten und zu analysieren. Denn letztlich stellt sich die Frage, wie wir leben und arbeiten wollen, was "gute Arbeit“ in einer europäischen und globalen Gesellschaft bedeutet.

\section{AUTOREN}

HARTMUT KLEIN-SCHNEIDER, Diplom-Volkswirt, Referatsleiter Betriebliches Personal- und Sozialwesen in der Abteilung Mitbestimmungsförderung der Hans-Böckler-Stiftung. Arbeitsschwerpunkte: Beschäftigungsverhältnisse, Nachtschichtarbeit, Flexible Arbeitszeit, Leistungsbezogenes Entgelt, Personalstrategien, unternehmensinterne Arbeitsmärkte.

hartmut-klein-schneider@ boeckler.de

KAI BEUTLER, Dipl.-Wirtsch.-Ing., Berater und Geschäftsführer bsb GmbH Betriebsräte-Strategie-Beratung Köln. Arbeitsschwerpunkte: Betrieblicher Arbeitsschutz und -gestaltung, Alternativen zu Leiharbeit und Werkvertragsarbeit, BR-Beteiligungsstrategien, "Gute Arbeit“, "Besser statt billiger“, Früherkennung und Bewältigung von Unternehmenskrisen.

@ kai.beutler@bsb-seite.de 